\title{
Space of Fontana
}

National Cancer Institute

\section{Source}

National Cancer Institute. Space of Fontana. NCI Thesaurus. Code C33579.

Any of the irregularly shaped spaces of the iridocorneal angle within the trabecular

reticulum that communicate with the sinus venosus sclera. 